Original

\title{
Novedades etiopatogénicas en el carcinoma de células renales: carcinogénesis y vias de desarrollo tumoral
}

\author{
Ernesto Sánchez Sánchez, Jesús Castiñeiras Fernández \\ Servicio de Urología. Hospital Regional Virgen Macarena. Sevilla. España
}

\begin{abstract}
Resumen
El cáncer renal representa el tercer tumor urológico más frecuente después del cáncer de próstata y vejiga. La incidencia anual se ha incrementado en los últimos años de forma notoria, en todos los estadios, lo cual implica un verdadero aumento del número de casos reales, no justificado plenamente por el uso masivo de pruebas de imagen diagnósticas.

Este trabajo pretende ser una actualización de la carcinogénesis y vías de desarrollo tumoral implicadas en la génesis de esta neoplasia. El carcinoma de células renales (CCR) se origina del epitelio renal y representa más del 90\% de todas las neoplasias malignas de este órgano. Aproximadamente el $2 \%$ de los CCR se asocian con síndromes hereditarios, oncogenes específicos o alteraciones de genes supresores tumorales. Todos los casos hereditarios presentan alteraciones del gen VHL. Este gen (que se localiza en el brazo corto del cromosoma 3:3p25-26), está también implicado en más del 60\% de los casos esporádicos. En este trabajo desarrollamos de forma sistemática los últimos avances sobre las implicaciones del gen VHL en la angiogénesis y su posible relación con las nuevas moléculas implicadas en el tratamiento del CCR.
\end{abstract}

Palabras clave: Cáncer renal. Carcinogénesis. Angiogénesis.

\section{New etiopathogenic findings in renal cell carcinoma: carcinogenesis and tumor development pathways \\ Abstract}

Renal cancer is the third leading urological tumor after prostate and bladder cancers. Annual incidence of renal cancer in all stages has markedly increased in recent years. This represents a true increase in the number of actual cases that is not fully accounted for by widespread use of diagnostic imaging tests.

This article is intended to provide an update on the carcinogenesis and tumor development pathways involved in the genesis of this tumor. Renal cell carcinoma (RCC) arises in renal epithelium and accounts for more than $90 \%$ of all malignant kidney tumors. Approximately 2\% of RCCs are associated to hereditary syndromes, specific oncogenes, or changes in tumor suppressor genes. Changes in the VHL gene exist in all hereditary cases. This gene (located in the short arm of chromosome 3:3p25-26) is also involved in more than 60\% of sporadic cases. This paper systematically addresses the latest findings on implications of the VHL gene in angiogenesis and its potential relationship to new molecules involved in management of RCC.

Keywords: Renal cancer. Carcinogenesis. Angiogenesis.

\section{CÁNCER RENAL: INCIDENCIA Y EPIDEMIOLOGÍA}

El cáncer renal representa el tercer tumor urológico más frecuente después del cáncer de próstata y vejiga. En Estados Unidos es el $7^{\mathrm{a}}$ tumor maligno con mayor incidencia entre los varones y el decimosegundo entre las mujeres ${ }^{1}$. En este país anualmente son diagnosticados 36.160 nuevos casos, y se han confirmado como posibles factores de riesgo conocidos, la edad, la obesidad, el fumar cigarrillos, el uso de ciertos fármacos (analgésicos, diuréticos...), la insuficiencia renal en estadios avanzados y la existencia de anormalidades renales tales como el riñón en herradura o la enfermedad renal quística adquirida ${ }^{2}$.
La incidencia anual se ha incrementado en los últimos años de forma notoria, en todos los estadios, lo cual implica un verdadero aumento del número de casos reales, no justificado plenamente por el uso masivo de pruebas de imagen diagnósticas ${ }^{3}$. De hecho, la proporción de pacientes que presentan metástasis en el momento del diagnóstico, no se ha modificado en los últimos años, lo cual justifica la existencia de un mayor número de casos y no meramente un adelanto diagnostico ${ }^{4}$.

En nuestro medio, la mortalidad de este tumor se ha mantenido estable en los últimos diez años. Según los datos publicados por el Instituto Nacional de Estadística (INE) ${ }^{5}$, la mortalidad se ha incrementado 
a la par que ha ido aumentando la población, por lo que la Tasa de Mortalidad (TM) ajustada ha variado sólo de 4,8/100.000 varones en 1996 a 5,04/100.000 varones en el año 2005 (Tabla 1). Los datos de TM en mujeres tampoco han experimentado variaciones significativas. Estos datos son consistentes y muy similares a los publicados recientemente por Levi ${ }^{6}$, el cual indica que la mortalidad por cáncer renal en Europa es del 4,1/100.000 varones en el periodo 2000-2004 y 1,8/100.000 mujeres en el mismo periodo.

\section{CÁNCER RENAL: ETIOPATOGENIA Y CLASIFICACIÓN OMS}

El carcinoma de células renales (CCR) se origina del epitelio renal y representa más del 90\% de todas las neoplasias malignas de este órgano. Aproximadamente el $2 \%$ de los CCR se asocian con sindromes hereditarios, oncogenes específicos o alteraciones de genes supresores tumorales ${ }^{7}$ (Tabla 2).
La clasificación de la Organización Mundial de la Salud (2004) distingue 3 subtipos histológicos fundamentales de tumores de células renales: convencional (células claras), papilar y cromófobo. Otros tipos más infrecuentes son: carcinoma de los conductos de Bellini, carcinoma medular, carcinoma Xp1 1, carcinoma asociado a neuroblastoma y carcinoma mucinoso tubular. Adicionalmente se ha introducido como una nueva categoría diagnóstica el CCR inclasificado, el cual incluye todos aquellos tumores que no se pueden encuadrar en ninguna otra categoría morfológica ${ }^{8}$ (Tabla 3 ).

\section{CCR CÉlULAS CLARAS}

El CCR, el cual es la variante histológica más frecuente, es un tumor maligno compuesto de células con citoplasma claro o eosinofilico. Representa el 70-85\% de los tumores diagnosticados en nuestro medio ${ }^{9-10}$. Normalmente se presenta como un tumor solitario

Tabla 1. Número total de defunciones en España por los principales tumores Urológicos.

Tasa de mortalidad (TM) ajustada a población.

\begin{tabular}{|c|c|c|c|c|c|c|}
\hline & \multicolumn{2}{|c|}{ C. Próstata (CP) } & Ca. vesical (CV) & Ca. testículo (CT) & \multicolumn{2}{|c|}{ C. Renal (CR) } \\
\hline 1996 & \multicolumn{2}{|c|}{5.459} & 3.436 & 44 & \multicolumn{2}{|c|}{1.582} \\
\hline 1997 & \multicolumn{2}{|c|}{5.480} & 3.746 & 49 & \multicolumn{2}{|c|}{1.682} \\
\hline 1998 & \multicolumn{2}{|c|}{5.728} & 3.697 & 33 & \multicolumn{2}{|c|}{1.624} \\
\hline 1999 & \multicolumn{2}{|c|}{5.638} & 3.913 & 37 & \multicolumn{2}{|c|}{1.573} \\
\hline 2000 & \multicolumn{2}{|c|}{5.448} & 3.909 & 43 & \multicolumn{2}{|c|}{1.674} \\
\hline 2001 & \multicolumn{2}{|c|}{5.651} & 4.103 & 44 & \multicolumn{2}{|c|}{1.694} \\
\hline 2002 & \multicolumn{2}{|c|}{5.664} & 4.195 & 41 & \multicolumn{2}{|c|}{1.644} \\
\hline 2003 & \multicolumn{2}{|c|}{5.597} & 4.287 & 47 & \multicolumn{2}{|c|}{1.806} \\
\hline 2004 & \multicolumn{2}{|c|}{5.677} & 4.493 & 40 & \multicolumn{2}{|c|}{1.808} \\
\hline \multirow[t]{2}{*}{2005} & & & 4.426 & 52 & & \\
\hline & $\mathbf{C P}$ & CV Varón & CV mujer & CT & CR Varón & CR Mujer \\
\hline TM 1996 & 24,16 & 12,91 & 1,73 & 0,21 & 5,04 & 1,75 \\
\hline TM 2005 & 18,22 & 13,10 & 1,79 & 0,20 & 4,8 & 1,68 \\
\hline
\end{tabular}

Fuente Instituto nacional de Estadística. http://www.ine.es/inebmenu/mnu_salud.htm.

Tabla 2. Alteraciones genéticas más frecuentes detectadas en los carcinomas de células renales.

\begin{tabular}{|c|c|c|c|}
\hline Sindrome & Gen & Alteración cromosómica & Aspecto patológico \\
\hline Von Hoppel Lindau & VHL & $3 p 25$ & $\begin{array}{l}\text { Múltiple, CCR bilateral, feocromocitoma, } \\
\text { hemangioblastoma }\end{array}$ \\
\hline $\begin{array}{l}\text { Carcinoma papilar renal hereditario } \\
\text { (CPRH) }\end{array}$ & MET & $7 q 31$ & $\begin{array}{l}\text { Múltiple, bilateral } \\
\text { CPRH tipo } 1\end{array}$ \\
\hline $\begin{array}{l}\text { Leiomiomatosis hereditaria y CCR } \\
\text { Leiomioma uterino. }\end{array}$ & $\mathrm{FH}$ & $1 q 42-43$ & CPRH no tipo 1 \\
\hline Síndrome Birt-Hogg-Dubé & $\mathrm{BDH}$ & 17911.2 & $\begin{array}{l}\text { Oncocitomas, tumores piel, CCR } \\
\text { múltiple }\end{array}$ \\
\hline $\begin{array}{l}\text { Carcinoma renal de células claras } \\
\text { hereditario }\end{array}$ & & 3p traslocación & Múltiple, CCR bilateral \\
\hline
\end{tabular}

Corti B, Zucchini N, Fabbrizio B, Martorana G et al. Pathology and molecular pathogenesis of renal cell carcinoma. Eur Urol Sup. 2006;5(8):573-579. 
Tabla 3. Clasificación de la OMS de los CCR

Tumores de células renales

Carcinoma de células claras (convencional)

Carcinoma de células claras multilocular

Carcinoma papilar

Carcinoma cromófobo

Carcinoma de conductos colectores de Bellini

Carcinoma medular renal

Carcinoma con translocación Xp11

Carcinoma asociado con neuroblastoma

Carcinoma mucinoso tubular y de células fusiformes

Carcinoma de células renales inclasificable

Adenoma papilar

Oncocitoma

Tumor inclasificable

Tumores metanéfricos

Adenoma metanéfrico

Adenofibroma metanéfrico

Tumor estromal metanéfrico

Tumores nefroblásticos

Restos nefrogénicos

Nefroblastoma (y nefroblastoma quístico parcialmente diferenciado)

Tumores mesenquimales principalmente pediátricos Sarcoma de células claras

Tumor rabdoide

Nefroma mesoblástico congénito

Tumor renal osificante de la infancia

Tumores mesenquimales principalmente de adultos Leiomiosarcoma (incluyendo los de la vena renal)

Angiosarcoma - Rabdomiosarcoma - Histiocitoma fibroso maligno

Hemangiopericitoma - Osteosarcoma - Leiomioma Hemangioma

Angiomiolipoma y angiomiolipoma epitelioide Linfangioma

Tumor de células yuxtaglomerulares - Schwanoma

Tumor renomedular de células intersticiales (fibroma medular)

Tumor fibroso solitario

Tumores mixtos epiteliales y mesenquimales

Nefroma quístico

Tumor mixto epitelial y estromal

Sarcoma sinovial

Tumores neuroendocrinos

Carcinoide

Carcinoma neuroendocrino

Tumor neuroectodérmico primitivo

Neuroblastoma

Feocromocitoma

Tumores hematopoyéticos y linfoides

Linfoma

Leucemia

Plasmocitoma

Tumores de células germinales

Teratoma

Coriocarcinoma

Tumores metastásicos riñón cortical que protuye hacía afuera de la corteza renal. Los tumores multicéntricos o bilaterales en estadios precoces son típicos de los síndromes hereditarios. Microscópicamente tienen un típico aspecto amarillento debido al rico contenido lipídico de sus células. Son frecuentes los quistes, hemorragias y calcificaciones dentro del tumor. Ocasionalmente puede presentar necrosis, lo cual implica un peor pronóstico clínico $^{11}$. Microscópicamente puede presentar una arquitectura alveolar, acinar o sólida. Normalmente suele presentar una tupida red vascular. El citoplasma de las células tumorales es habitualmente claro. El núcleo puede ser redondeado o poligonal con nucleolos y una cromatina finamente distribuida. El pleomorfismo nucleolar, los nucleolos prominentes son hallazgos típicos de los tumores de alto grado.

El CCR de células claras puede ser una manifestación de la enfermedad de von Hippel-Lindau en asociación a otros tumores extrarrenales. Todos los casos hereditarios presentan alteraciones del gen VHL. Este gen (que se localiza en el brazo corto del cromosoma 3: 3p25-26), está también implicado en más del 60\% de los casos esporádicos. Para que este gen pierda su capacidad supresora es necesario la pérdida de sus dos alelos, lo cual puede suceder por tres mecanismos: pérdida de su carácter heterozigótico (84-98\% de los casos), mutaciones (34-57\% casos) y metilación de la región promotora del gen (5-19\% casos). Aunque la supresión del gen VHL es un factor de riesgo importante para el posible desarrollo de CCR de células claras, el 30-40\% de las formas esporádicas no muestran alteraciones de dicho gen. Cuando este VHL es introducido en cultivos celulares de CCR deficientes de gen VHL, el crecimiento del tumor era inhibido tanto in vivo como in vitro $^{12}$. Las diferencias biológicas y de comportamiento clínico de estas células tumorales sugieren que posiblemente haya un único subconjunto de CCR de células claras ${ }^{13}$.

La función del gen VHL es codificar la proteína VHL (pVHL), componente crítico de una secuencia celular responsable del aporte de oxígeno a través de la regulación de la trascripción de un factor llamado Factor Inducible por Hipoxia (HIF: hipoxiainducible-factor). El HIF es un factor de trascripción heterodimérico (HIF $\alpha / \beta)$ encargado de facilitar la adaptación del tejido a la hipoxia. La proteína VHL (pVHL) se encarga de la degradación de la HIF $\alpha$, 
especialmente cuando el oxígeno es abundante. A diferencia de las células normales, las células con déficit de VHL acumulan inapropiadamente HIF $\alpha$ en condiciones de normal oxigenación tisular, lo cual conlleva una sobreproducción del factor de crecimiento del endotelio vascular (VEGF), factor de crecimiento $\beta$ derivado de plaquetas (PDGF $\beta$ ) y factor transformante del crecimiento a (TGF- $\alpha$ ). Esta sobreregulación del HIF en las células deficitarias de VHL juega un papel fundamental en la tumorogénesis. Se ha comprobado que la capacidad de las células de tumores renales deficitarias de VHL de formar tumores puede reducirse significativamente por la inactivacion del $\mathrm{HIF}^{14}$.

En principio, la interrupción de la producción de estos factores de crecimiento y factores angiogénicos podría privar al tumor de elementos que contribuyen a su propia supervivencia. Un ejemplo de este mecanismo de acción lo podemos comprender con el uso de bevacizumab, un anticuerpo monoclonal dirigido contra el VGEF. En un ensayo clínico fase 2, randomizado y controlado con placebo, en pacientes con CCR metastático tratados con alta dosis de bevacizumab, se demostró una respuesta parcial en un $10 \%$ de los pacientes, y hubo una mejoría estadísticamente significativa en la progresión libre de enfermedad $^{15}$.

Sunitinib y Sorafenib inhiben la vía del VEGF y PDGF $\beta$, bloqueando el receptor 2 de la VEGF (VEGFR2) y el receptor $\beta$ del PDGF (PDGFR $\beta$ ), los cuales son receptores tirosin-kinasas. El bloqueo del receptor TGF- $\alpha$ (EGFRR) no ha demostrado, hasta el momento actual, la misma eficacia clínica ${ }^{16}$ (Figura 1).

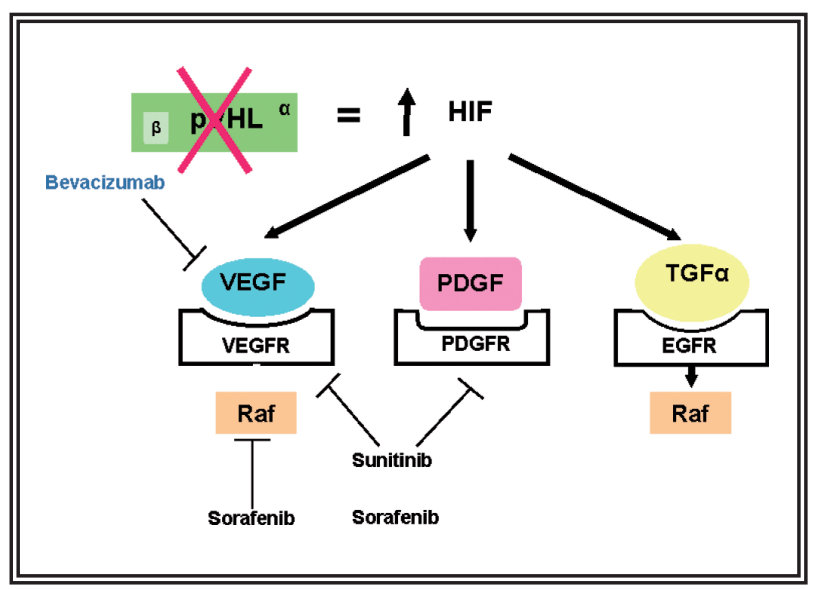

FIGURA 1. Dianas terapéuticas en carcinomas de células renales. Proliferación celular y angiogénesis.
Basados en los resultados descritos anteriormente, Rini et al. ${ }^{17}$ propusieron la teoría de que la existencia de mutaciones o metilaciones, pudiera ser utilizada como un factor pronóstico de la respuesta de los pacientes a estos tratamientos antiangiogénicos. Cuarenta y tres pacientes fueron evaluados, observándose respuestas objetivas tras el tratamiento en el $48 \%$ de los pacientes con alteraciones del gen VHL. Estos pacientes presentaban una mediana de tiempo a la progresión del tumor de 13,3 meses, frente a 7,4 meses en aquellos pacientes sin las mencionadas alteraciones del gen VHL.

\section{Expresión anhidrasa carbónica}

La anhidrasa carbónica IX (CAIX), juega un papel fundamental en el mantenimiento del $\mathrm{PH}$ intracelular de la célula tumoral en condiciones de hipoxia o glicólisis intensa. La expresión de CAIX es aceptada de forma unánime como un marcador de regiones hipóxicas. Debido a que la expresión de CAIX se ha observado en la mutación de VHL asociado con CCR papilar tipo I, ha existido un creciente interés en su mecanismo de acción para desarrollar posibles agentes terapéuticos ${ }^{18}$.

Bui et al. ${ }^{19}$ demostraron que los pacientes con CCR avanzado que tenían una baja expresión de CAIX tenían un peor pronóstico. Lo resultados también demostraron que una elevada expresión del CAIX se relacionaba con una mayor supervivencia y una mayor probabilidad de respuesta al tratamiento con interleukina 2 .

Posteriormente Sandlund et al. ${ }^{20}$ en 2007, corroboraron el valor pronóstico del nivel de CAIX en CCR de células claras. Los resultados del estudio demostraron que la expresión de CAIX era significativamente mayor $(\mathrm{p}<0,001)$ en CCR convencional versus CCR papilar o cromófobo. En el CCR convencional no se demostró correlación de la expresión de CAIX con el estadio TNM o grado nuclear. Los pacientes que expresaban CAIX en cantidades inferiores al $10 \%$ tenían un pronóstico menos favorable que aquellos con expresión del 11 al 90\% (p=0,012) o aquello con expresión del 91 al 100\% ( $\mathrm{p}=0,001)$. Aunque este estudio confirma los resultados obtenidos por BUI et al., estas diferencias pronósticas se encontraron sólo en pacientes con enfermedad en estadio I-III, lo cual contrasta con los resultados previos, que demostraban solo diferencias pronósticas en pacientes con estadio IV. 
Recientemente Pantuck et al. ${ }^{21}$ presentaron un estudio para intentar descubrir la conexión entre CAIX, la mutación del gen VHL y las características del tumor. Sus resultados demostraron que la baja expresión de CAIX se asociaba con la falta de mutación del gen VHL y mayor agresividad biológica del tumor y era un factor estadísticamente significativo de peor pronóstico en pacientes con CCR de células claras. El 58\% de los pacientes presentaban mutaciones del gen VHL y la expresión de CAIX osciló desde el 0 al 100\%. Una expresión de CAIX inferior al $85 \%$ se asociaba a la ausencia de mutación del gen VHL, tumores de mayor tamaño, mayor estadio $\mathrm{T}$, metástasis linfáticas y mayor grado de Fuhrman. Además, la ausencia de mutación del gen VHL se asociaba con la presencia de adenopatías y metástasis a distancia. Los pacientes con altos niveles de CAIX y mutación del VHL presentaban mejores supervivencias $(95,9 \%$ al primer año y mediana de supervivencia de 6 años) frente a los pacientes con baja expresión de CAIX y ausencia de mutación de VHL (62\% supervivencia al año y mediana de supervivencia de 1,5 año).

Hasta el momento actual no se ha estudiado el papel de los niveles de CAIX en la respuesta a los nuevos tratamientos antiangiogénicos ${ }^{22}$. El papel de la sobreexpresión de gen p53 está aún en debate. Sólo unos pocos estudios demuestran una asociación claramente significativa ${ }^{23}$. Se ha demostrado una asociación significativa entre el descenso de los niveles de expresión del gen p27 y el aumento del tamaño tumoral y grado de Fuhrrman ${ }^{24}$. Sin embargo, no se ha demostrado una asociación entre la disminución de la expresión de este marcador y la supervivencia.

\section{OTROS TIPOS DE CCR CÉLULAS CLARAS CCR tipo papilar}

Se define por la distribución de sus células alrededor de ejes capilares (papilas) al menos en un 50$70 \%$ del tumor, diferenciándose así de las ocasionales áreas papilares que pueden hallarse en otros tipos de carcinoma renal. Un $73 \%$ de los casos está formado por células basófilas (Tipo 1) y un 42\% de células eosinófilas (Tipo 2). Los primeros suelen tener bajo grado celular y mejor pronóstico que los Tipos 2. Su incidencia es variable pero pueden llegar a ser del 7 al 15\% de los CCR.

Los cambios genéticos más frecuentes son la trisomía o tetrasomía del 7 , trisomía 17 y pérdida del cromosoma Y. Estas alteraciones se han relacionado con la activación del proto-oncogen c-MET (7q34) que codifica el receptor de HGF (rHGF). También se ha detectado pérdida de heterogeneidad $3 p$ en un $59 \%$ de los $\operatorname{casos}^{25}$. Recientemente se ha encontrado que las alteraciones en $17 q$ son casi exclusivas del Tipo 1, mientras que las 9p son del Tipo 2, 10 que sugiere que cada uno de estos tipos celulares puede surgir de diferentes tipos celulares ${ }^{26}$.

\section{CCR de células tipo cromófobo}

Representa del 5 al 10\% de los CCR en nuestro medio.

Los cambios moleculares más frecuentemente encontrados son pérdida en los cromosomas 1, 2, 6, $10,10,17$, y 21 . Las pérdidas de heterogeneidad del cromosoma 17 lo relacionan con el Sindrome de Birt-Hogg-Dubé.

\section{CONCLUSIONES}

Claramente se puede afirmar que en los últimos años se han hecho avances considerables en la comprensión y clasificación de los tipos de CCR. El uso de la histopatología es útil por su gran valor diagnóstico y pronóstico. Aunque el resultado de los distintos estudios presentados puede ser contradictorio en algunos aspectos, se confirma que hay una necesidad de estudios futuros para confirmar los hallazgos descubiertos y ofrecer luz sobre los aspectos aun controvertidos. La evidencia de que existen conexiones claras entre la mutación VHL y la expresión de CAIX puede ser usada como un marcador pronóstico para predecir la eficacia de distintos tratamientos en pacientes con CCR.

\section{REFERENCIAS}

1. Jemal A, Murray T, Ward E, Samuels A, Tiwari RC, Ghafoor A,, et al. Cancer Statistics, 2005. CA cancer J Clin. 2005;55(1):10-30.

2. Chow WH, Devesa S, Warren J, Fraumeni JF Jr. Rising incidence of renal cell cancer in the United States. JAMA. 1999;281(17): 1628-1631.

3. Hock LM, Lynch J, Balaji KC. Increasing incidence of all stages of kidney cancer in the last decades in the United States: an analysis of surveillance, epidemiology and end results program data. J Urol. 2002;167(1);57-60.

4. Rubaggotti A, Martorana G, Boccardo F. Epidemiology of kidney cancer. Eur Urol Supp. 2006;5(8):558-565.

5. Instituto Nacional de Estadística de España. (INE). 2009. http://www.ine.es/inebmenu/mnu_salud.htm.

6. Levi F, Ferlay J, Galeone C, Lucchini F, Negri E, Boyle P, et al. The changing pattern of kidney cancer incidence and mortality in Europe. BJU Int. 2008;101(8):949-958.

7. Corti B, Zucchini N, Fabbrizio B, Martorana G. Pathology and molecular pathogenesis of renal cell carcinoma. Eur Urol Sup. 2006;5(8):573-579 
8. Eble JN, Togashi K, Pisani P. Renal cell carcinoma. In: Eble JN, Sauter G, Epstein JI, Sesterhenn IA, editors. Pathology and genetics of tumors of the urinary system and male genital organs. World Health Organization Classification of Tumors. Lyon: IARC Press; 2004;12-43.

9. Fleming S, O’Donell M. Surgical pathology of renal epithelial neoplasms: recent advances and current status. Histopathology. 2000;36(3): 195-202

10. Patard JJ, Leray E, Rioux-Leclercq N, Cindolo L, Ficarra V, Zisman A, et al. Prognostic value of histologic subtypes in renal cell carcinomas: a multicenter experience. J Clin Oncol. 2005; 23(12):2763-2771.

11. Sengupta S, Lohse CM, Leibovich BC, Frank I, Thompson RH, Webster WS, et al. Histologic coagulative necrosis as a prognostic indicator of renal cell carcinoma aggressiveness. Cancer. 2005; 104(3):511-520.

12. Yao M, Yoshida M, Kishida T, Nakaigawa N, Baba M, Kobayashi $\mathrm{K}$, et al. VHL tumor suppressor gene alterations associated with good prognosis in sporadic clear-cell carcinoma. J Nat Cancer Inst. 2002;94(20):1569-1575.

13. Parker AS, Cheville JC, Lohse CM, Igel T, Leibovich BC, Blute ML. Loss of expression of von Hippel Lindau tumor suppressor protein associated with improved survival in patients with early stage clear cell renal cell carcinoma. Urology. 2005;65(6):1090-1095.

14. Zimmer M, Doucette D, Siddiqui N, Iliopoulos O. Inhibition of hipoxia-inducible factor is suffcient for growth supression of VHL-/- tumor. Mol Cancer Res. 2004;2(2):89-95.

15. Yang JC, Haworth L, Sherry RM, Hwu P, Schwartzentruber DJ, Topalian SL, et al. A randomized trial of bevacizumab, an antivascular endothelial growth factor antibody, for metastatic renal cancer. N Engl J Med. 2003;349(5):427-434.

16. Brugarolas J. Renal-cell carcinoma-molecular pathways and therapies. N Engl J Med. 2007;356(2):185-7.

17. Rini BI, Jaeger E, Weinberg V, Sein N, Chew K, Fong K, et al. Clinical response to therapy targeted at vascular endotelial growth factors in metastatic renal cell carcinoma: impact of patient characteristics and Vonm Hippel-Lindau status. BJU Int. 2006;98(4):756-762.

18. Dorai T, Sawczuck I, Pastorek J, et al. Role of carbonic anhidrases in the progression of renal cell carcinoma subtypes: proposal of an unified hypothesis. Cancer Invest 2006; 34: 754-779.
19. Bui MH, Seligson D, Han KR, Pantuck AJ, Dorey FJ, Huang Y, et al. Carbonic anhidrase IX is an independent predictor of survival in advanced renal clear cell carcinoma: implications for prognosis and therapy. Clin Cancer Res. 2003;9(2):802-811.

20. Sandlund J, Oosterwijk E, Grankvist K, Oosterwijk-Wakka J, Ljungberg B, Rasmuson T. Prognostic impact of carbonic anhydrase IX expression in human renal cell carcinoma. BJU Int. 2007;100(3):556-560.

21. Pantuck AJ, Trinh Q, Karakiewicz PI, et al. Use of carbonic anhydrase IX (CAIX) expression and von Hippel-Lindau (VHL) gene mutation status to predict survival in renal cell carcinoma (Abstract 5042). Presented at the 2007 ASCO Annual Meeting. Chicago, Ill: June 1-5, 2007.

22. Patard JJ, Fergelot P, Karakiewicz PI et al. Low CAIX expression and absence of VHL gene mutation are associated with tumor aggressiveness and poor survival of clear cell renal cell carcinoma. Int J Cancer. 2008 Jul 15;123(2):395-400.

23. Uchida T, Wada C, Shitara T, Egawa A, Mashimo S, Koshiba K. Infrequent involvement of p53 mutations and loss of heterozygosity of $17 \mathrm{p}$ in the tumorigenesis of renal cell carcinoma. $\mathrm{J}$ Urol.1993; 150(4):1298-1301.

24. Schiavina R, Manferrari F, Bertaccini A, et al. Valore Prognostico della p27 nei Tumori Renali: Studio Clinico-Patologico. Abstracts XIII Congresso Nazionale Societa` taliana di Urologia Oncologica. Arch Ital Urol Androl 2003;75:49.

25. Algaba F, Arce Y, Trias I, Santaularia JM, Rosales A. Aplicaciones clínicas de las actuales clasificaciones del cáncer renal. Act Urol Esp. 2006;30:372-385.

26. Sanders ME, Mick R, Tomaszewski JE, Barr FG. Unique patterns of allelic imbalance distinguish type 1 from type 2 sporadic papillary renal cell carcinoma. Am J Pathol. 2002;161(3): 997-1005.

Correspondencia autor: Dr. Ernesto Sánchez Sánchez Servicio de Urología. Hospital Regional Virgen Macarena. Sevilla Av. Dr. Fedriani s/n - 41071 Sevilla. Tel.: 955008265 E-mail autor: ernesto@pulso.com Información artículo: Original Trabajo recibido: abril 2009

Trabajo aceptado: mayo 2009 